\title{
PERSPECTIVAS DE DESARROLLO DE LA INDUSTRIA SEMILLERA DE MAÍZ EN MÉXICO
}

\author{
PERSPECTIVES OF THE MAIZE SEED INDUSTRY DEVELOPMENT IN MÉXICO
}

\author{
Bethel M. Luna Mena ${ }^{1}$, Ma. Alejandra Hinojosa Rodríguez ${ }^{2}$, Óscar J. Ayala Garay ${ }^{1 \star}$, Fernando Castillo \\ González $^{1}$ y J. Apolinar Mejía Contreras ${ }^{1}$
}

\begin{abstract}
${ }^{1}$ Postgrado en Recursos Genéticos y Productividad, Colegio de Postgraduados-Campus Montecillo. Km 36.5 carretera México-Texcoco. 56230, Montecillo, Texcoco, Estado de México. ${ }^{2}$ División de Ciencias Económico Administrativas, Universidad Autónoma Chapingo. Km. 38.5 carretera México-Texcoco. 56230, Chapingo, Estado de México.
\end{abstract}

*Autor para correspondencia (oayala@colpos.mx)

\section{RESUMEN}

La producción y distribución de semillas mejoradas son el puente de transferencia de tecnología entre fitomejoradores y productores, para alcanzar niveles competitivos en la producción. Sin embargo, en México las semillas mejoradas de maíz (Zea mays L.) se siembran en sólo $30 \%$ de la superficie ocupada por este cultivo. En esta investigación se estudió el mercado nacional de semillas y se analizó la posibilidad de desarrollo de pequeñas empresas productoras de semilla de maíz. Con la Ley de Producción, Certificación y Comercio de Semillas (LPCCS) de 1961 se permitió al Estado Mexicano promover la generación de variedades y la producción y comercio de semillas. En 1989 se produjeron 350 mil toneladas de semilla, de las cuales 28 mil fueron de maíz. En 1991 se promulgó una nueva LPCCS que permitió la participación sin restricciones del sector privado en la investigación, producción y comercialización de semilla, y también se restringió al sector público al grado de que la Productora Nacional de Semillas fue liquidada. Esto originó una fuerte caída en la producción de semillas ( 163 mil toneladas en 1995, de las cuales 15 mil fueron de maíz) y la expansión masiva de megaempresas transnacionales en la industria semillera nacional, que han consolidado un oligopolio. En 2009 se sembraron en México 8 millones de hectáreas de maíz, de las cuales 1.5 millones fueron con 45 mil toneladas de semilla híbrida. De éstas, 95 $\%$ fueron producidas por Monsanto y Pioneer principalmente, y el resto ( 3 mil toneladas) por pequeñas empresas. Aquí se propone promover la participación de pequeñas empresas productoras de semilla de maíz a escala local y regional, que atiendan a productores que siembran superficies reducidas en regiones agroecológicas especiales, lo cual ampliaría la superficie sembrada con semilla mejorada. Esta acción se debe articular con una dinámica agrícola (cadena de valor) que genere nuevas variedades y atienda la producción, distribución, asesoría técnica y comercialización del maíz.

Palabras clave: Zea mays, producción de semilla, historia de la investigación agrícola, mercado de semillas.

\section{SUMMARY}

The production and distribution of improved seeds are the link for technology transfer between plant breeders and farmers, to achieve competitive levels of production. However, in México only $30 \%$ of the agricultural land is planted with improved maize (Zea mays L.) varieties. In this research, the domestic maize seed market was analyzed regarding the opportunities of development of small enterprises for seed production. The Production, Certification and Seed Trade Bill (PCSTB) published in 1961, created an institutional framework that allowed the Mexican State to promote the generation of varieties, as well as seed production and its trade. Seed production reached 350000 tons in 1989, of wich 28000 were of maize. In 1991, a new PCSTB was published which allowed an unrestricted participation of the private sector on agricultural research, seed production and marketing, while the public sector became so resticted that the National Seed Producing Company was eliminated. This second bill resulted in a large reduction of seed production ( 163000 tons in 1995, of which 15000 were of maize) and the massive expansion of transnational mega-corporations in the national seed industry, which soon consolidated into an oligopoly. In 2009, 8 million hectares of maize were seeded in México, of which 1.5 million were planted with 45000 tons of hybrid seeds. Most of these seeds $(95 \%)$ were produced by Monsanto and Pioneer, and the rest by small companies (3000 tons). In this study we propose participation of the local and regional small companies to address small-scale farmers in specific agro-ecological regions, so that the area planted with improved seeds would expand. This approach must be articulated with a dynamic agricultural system (value chain) that would generate new maize varieties along with seed production, distribution, technical service and marketing.

Index words: Zea mays, seed production, history of agricultural research, seed market.

\section{INTRODUCCIÓN}

La viabilidad de la agricultura es fundamental para garantizar alimentos a la población. La seguridad regional y nacional alimentaria depende de varios factores, entre los que destacan el mantenimiento y fortalecimiento de un sistema de investigación y desarrollo de una manera incluyente, que sea capaz de proporcionar continuas mejoras en todos los aspectos de los sistemas productivos utilizados por los agricultores, que haga frente a la diversidad agroecológica, económica y social de un país, y que sea dinámica para responder a los cambios climáticos 
(Tansey y Rajotte, 2009).

El maíz (Zea mays L.) es el cultivo más importante de México, desde el punto de vista alimentario, económico, político y social. Este grano se produce en dos ciclos agrícolas: primavera-verano y otoño-invierno, bajo diversas condiciones agroclimáticas de humedad: secano (temporal), punta de riego y riego (SIAP, 2007).

Desde el punto de vista económico, el maíz se siembra en más de 8 millones de hectáreas, que representa $39 \%$ de la superficie agrícola nacional y $63 \%$ de la superficie sembrada con granos y oleaginosas; contribuye con $8 \%$ del producto interno de la agricultura y es el cultivo que más fuerza de trabajo ocupa. No obstante, se importaron 8 millones de toneladas de grano de maíz en 2006 y 10 millones de toneladas en 2010 , lo que pone a este alimento a la cabeza de las importaciones de productos agrícolas de México. El minifundio y la pequeña propiedad también predominan en este cultivo (González et al., 2008). De acuerdo con Espinosa et al. (2008), en años recientes este déficit se incrementó debido a la inadecuada estrategia gubernamental que prefería importar grano en lugar de producirlo, bajo el argumento de que el precio internacional en términos relativos era menor al costo nacional.

La adopción de mejores tecnologías es un requisito para que los productores de maíz mejoren su competitividad. Se ha estimado que el potencial de producción sostenible de este cereal en México es de 52 millones de toneladas, de las cuales 28 millones serían factibles de lograr en el corto plazo (tres a seis años), sin incrementar la superficie sembrada y sin utilizar maíz transgénico, mediante la aplicación de tecnología de producción, variedades y prácticas de cultivo disponibles, desarrolladas por instituciones públicas nacionales de investigación y de educación superior (Turrent-Fernández, 2009).

De acuerdo con Copeland y McDonald (2001), las semillas de variedades mejoradas son el medio para incrementar el rendimiento y calidad de las cosechas, al servir como puente entre el mejoramiento genético (la investigación) y el productor. En países en desarrollo el uso de semillas mejoradas permitiría alcanzar niveles competitivos en la producción. Sin embargo, en México su uso en maíz es escaso, pues se estima que es de 26 a $33 \%$ (Espinosa et al., 2003).

La adopción de semilla mejorada es un proceso de cambio, por lo que conviene que los asesores técnicos e investigadores agrícolas se familiaricen con los factores agroecológicos y sociales que intervienen en el proceso, para poder apoyar y orientar con eficiencia a los productores. Además, es conveniente que los agricultores reciban capacitación específica sobre las formas organizacionales más efectivas de tomar decisiones individuales y de grupo, ya que todo proceso de transferencia de tecnología involucra cargas afectivas y reflexivas precisamente en las etapas en que los individuos deben tomar decisiones (Guillén-Pérez et al., 2002).

La creciente compactación e integración mundial de los suministradores de insumos agrícolas, en la que cada vez menos empresas controlan el mercado, ha influido en el desarrollo del sistema alimentario. El control del mercado, cada vez más concentrado por medio de inversiones, fusiones y compras, permite que grandes consorcios impongan precios, reduzcan la competencia y garanticen su predominio dentro del sector. Por ejemplo, el mercado mundial de semillas mejoradas está ahora concentrado en media docena de empresas que mantienen la exclusividad de un número considerable de variedades. Además, el control de estas empresas sobre la propiedad intelectual del germoplasma y las redes de distribución de semillas ha aumentado las barreras que impiden que nuevas firmas entren en el campo de desarrollo de germoplasma, lo que presagia una convergencia sin precedentes entre los segmentos clave del mercado agrícola: productos agroquímicos, semillas, tecnología agrícola tradicional y biotecnología (Tansey y Rajotte, 2009).

En 2011, a 50 años de la publicación de la primera Ley de Semillas (DOF, 1961) en México, y con la puesta en marcha del Programa de Modernización Sustentable de la Agricultura Tradicional (MasAgro), destaca nuevamente la importancia de la industria nacional de semillas, sobre todo de las empresas locales y regionales, como agentes básicos del programa, cuyo objetivo principal es incrementar la producción nacional de maíz de la agricultura tradicional, en donde las variedades apropiadas para estas condiciones han sido desarrolladas por los organismos de investigación pública y no por las empresas transnacionales. La participación de pequeñas y medianas empresas productoras de semilla que multipliquen las variedades adaptadas a regiones específicas y ofrezcan a los productores de maíz semilla de calidad a precios accesibles, les permitiría cumplir con la función de instituciones clave que existían en el sector semillas, como la extinta Productora Nacional de Semillas (PRONASE).

El impacto actual en el comercio de semilla de las variedades mejoradas de maíz liberadas por el Instituto Nacional de Investigaciones Forestales, Agrícolas y Pecuarias (INIFAP) y por otras instituciones públicas, es limitado, a pesar de que existen materiales genéticos destacados. Una causa es la insuficiencia, en número y tamaño, de empresas productoras de semilla que multipliquen este insumo; además, su abastecimiento de semilla registrada no es 
adecuado ni oportuno, por lo que es frecuente que los planes de las empresas de semillas, usuarias del INIFAP, reduzcan sus programas de producción. Por ello se requiere propiciar y apoyar el desarrollo de empresas mexicanas productoras de semilla de maíz y de otras especies, para garantizar el abastecimiento de semilla certificada (González et al., 2008).

El objetivo de la presente investigación fue estudiar el mercado nacional de semillas para analizar las posibilidades de establecer y desarrollar pequeñas y medianas empresas productoras de semilla de maíz.

\section{ANTECEDENTES HISTÓRICOS DE LA PRODUCCIÓN DE SEMILLA DE MAÎ́Z EN MÉXICO}

La evolución histórica de la investigación agrícola y la consecuente producción de semilla mejorada, es determinante para entender la situación actual de la actividad semillera. En México, la investigación agrícola formal se inició con la creación del Colegio Nacional de Agricultura en 1853, y al año siguiente, el 22 de febrero, se estableció la Escuela Nacional de Agricultura y Veterinaria en San Jacinto, D. F., lo que dio inicio a la educación, investigación y divulgación agrícola, pecuaria y forestal. Este proceso se detuvo cuando la entonces Escuela Nacional de Agricultura suspendió actividades durante la Revolución Mexicana en 1910. En 1919 reinicia actividades y en el país se impulsan programas de fomento agrícola, en los que el reparto de tierras era prioritario. Se establecieron también los programas denominados de propaganda agrícola, así como las primeras estaciones agrícolas experimentales ubicadas en Yucatán, Tabasco, San Luis Potosí, Chihuahua, y la estación central en San Jacinto (Jiménez-Sánchez, 1984).

En 1933 los campos agrícolas se agruparon en el Departamento de Campos Experimentales (DCE) dependiente de la Dirección General de Agricultura. Ahí se efectuaron investigaciones de tipo agronómico, así como algunas introducciones y pruebas de adaptación de variedades. Como tales introducciones no pudieron usarse por problemas de adaptación, se decidió iniciar programas de mejoramiento genético. La investigación se intensifica al establecerse en 1943 la Oficina de Estudios Especiales (OEE) con financiamiento de la Fundación Rockefeller, y el DCE evoluciona a Instituto de Investigaciones Agrícolas (IIA) en 1946. Las primeras especies en que se hizo investigación fueron maíz, trigo (Triticum aestivum L.) y frijol (Phaseolus vulgaris L.). Las primeras variedades mejoradas de maíz se liberaron en 1947, en 1948 las de trigo y en 1949 las de frijol. Para 1950 la OEE y el IIA habían liberado 23 variedades de maíz, 10 de trigo y tres de frijol (Ayala-Garay et al., 2006).
La disponibilidad de variedades mejoradas y la expectativa de lograr incrementos en la producción, propiciaron la creación de la Comisión del Maíz en 1947, con el propósito de multiplicar, promover y fomentar el uso de semilla mejorada de esta especie. En 1949, dado su crecimiento, se le reconoce como Comisión Nacional del Maíz (Ayala-Garay et al., 2006).

En 1954 se implementó un programa de emergencia para incrementar la producción agrícola, que propició se multiplicaran los campos de producción de semillas de variedades de los tres principales cultivos básicos. Ante la necesidad de establecer un control de calidad de semillas y su certificación, surgió el Departamento de Semillas de la Dirección de Agricultura. En 1958 se creó el Comité para la Producción y Distribución de Semillas Mejoradas. En 1959 se logró la autosuficiencia en grano de maíz, frijol y trigo. Por este éxito y dado que las necesidades de semillas de variedades mejoradas de buena calidad eran crecientes, en diciembre de 1960 se decretó la Ley sobre Producción, Certificación y Comercio de Semillas (LPCCS), publicada el 14 de abril de 1961 (DOF, 1961). Con base en consideraciones de orden técnico, económico, político y social se fusionaron la OEE y el IIA para dar origen al Instituto Nacional de Investigaciones Agrícolas (INIA). Igualmente, en ese año la Comisión Nacional del Maíz se convierte en la Productora Nacional de Semillas (PRONASE).

De esta manera, conforme a lo dispuesto por la LPCCS, se creó el Sistema Nacional de Producción, Certificación y Comercio de Semillas, integrado por el INIA, el Comité Calificador de Variedades de Plantas (CCVP), el Registro Nacional de Variedades de Plantas (RNVP), la PRONASE y el Servicio Nacional de Inspección y Certificación de Semillas (SNICS), responsable de vigilar los aspectos concernientes a la certificación y comercio de semillas (DOF, 1961).

En la Ley de Semillas de 1961 se establecía que el mejoramiento genético de plantas correspondía al INIA y que las semillas originales generadas se entregarían a la PRONASE para su aprovechamiento en los programas de producción de semillas en escala comercial. De esta manera, el Estado era el responsable de la producción y comercio de semillas, para lo que se disponía de un esquema institucional que atendía cada una de las funciones específicas del sistema agrícola del país.

El sector privado dedicado a la producción de semilla consideraba restrictiva esta Ley y comenzó a presionar para participar en aspectos relacionados con la investigación y el desarrollo de variedades mejoradas propias. En 1968 se crea la Asociación Mexicana de Semilleros, A. C. (AMSAC), 
uno de cuyos objetivos fue conseguir autorización para que sus asociados realizaran investigación, y obtuvieron el primero de ellos en 1976. Así, en 1983 se autorizó la venta de semilla de variedades mejoradas de maíz producto de la investigación privada en algunas áreas de México (AyalaGaray et al., 2006).

A principios de la década de los años de 1990, para adecuarse a las políticas de la globalización comercial, el gobierno de México hizo una serie de cambios en la legislación, que afectó directamente al sector agrícola. Así, se promulgó una nueva Ley de Semillas que permitió la participación sin restricciones al sector privado en la investigación agrícola, particularmente en la producción y comercialización de semillas (DOF, 1991). Además, se abrió la posibilidad de que otros usuarios multiplicaran y comercializaran semilla de variedades generadas por instituciones públicas, como las del Instituto Nacional de Investigaciones Forestales, Agrícolas y Pecuarias (INIFAP, antes INIA), además de la PRONASE.

Con esta Ley y la Ley Federal de Variedades Vegetales de 1996, se permitió la participación de la iniciativa privada, que aunado a políticas de inversión del sector público y presiones de organismos internacionales, originó que paulatinamente ocurriera el desmantelamiento de la PRONASE.

La entrada masiva de la iniciativa privada en la industria de semillas, constituida principalmente por empresas transnacionales, no garantizó elevar la producción de semilla certificada como se hubiera esperado. El abastecimiento de semillas se concentró en las regiones de mayor potencial productivo y en agricultores de mayores recursos económicos, pero se desatendieron las áreas de subsistencia y agricultura tradicional. El análisis de la producción de semilla en México (Figura 1), realizado según estadísticas del SNICS, muestra que en 1988 y hasta 1990 se produjeron más de 300 mil toneladas de materia prima de semilla certificada. Sin embargo, con la entrada en vigor de la Ley de Semillas de 1991, inicia una disminución en la producción de semilla. Esta tendencia permanece en los años siguientes, y aunque en ciertos años se ha elevado la producción tales cifras no superan en ningún año las 300 mil toneladas obtenidas al principio del periodo de análisis. En cuanto al maíz, las cantidades de semilla producida continuaron creciendo hasta dos años después de la promulgación de la ley, para después caer hasta un mínimo de 14.9 mil toneladas en 1995; vuelve a repuntar y en 1997 se produjeron 43.3 mil toneladas, y se obtuvieron incrementos posteriores con una tasa anual promedio de 2.5 mil toneladas hasta 2009 (Figura 1).

El surgimiento de pequeñas y medianas empresas nacionales y la participación de organizaciones de productores en la industria de semillas, fue restringido por la expansión de grandes empresas transnacionales, y al no existir mecanismos que regularan el crecimiento del sector privado, ni políticas que permitieran el desarrollo y crecimiento de la industria nacional, ocurrió un proceso de concentración en la producción y la comercialización de semilla, que redujo la capacidad de competir en igualdad de condiciones.

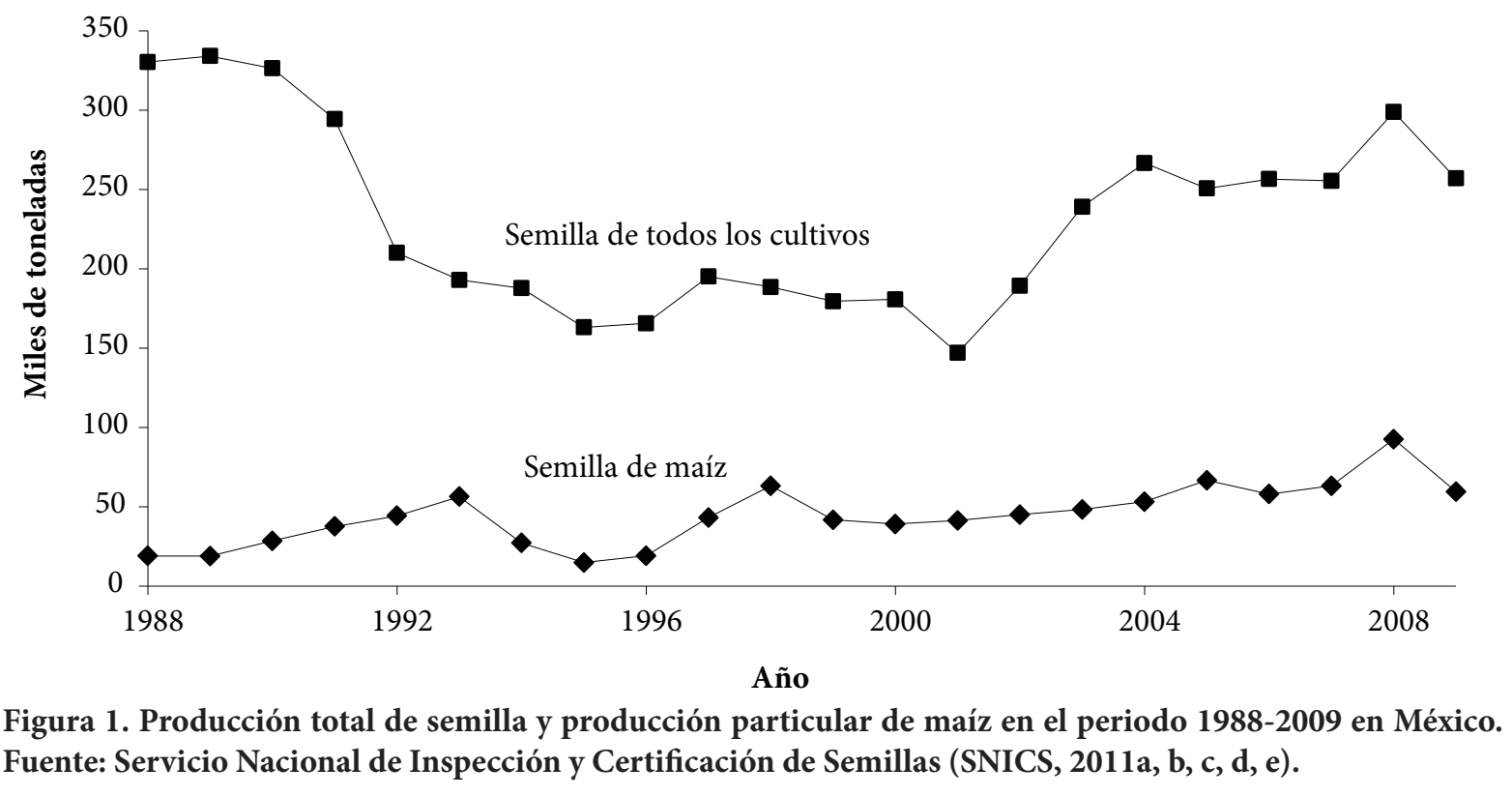




\section{MERCADO MUNDIAL DE SEMILLAS}

El mercado mundial de semillas se ha caracterizado por un proceso de fusión de empresas, intensificado en los años 1980. En 1985 (Cuadro 1), Pioneer encabezaba la lista de las empresas productoras de semillas y superaba en $39 \%$ el volumen de ventas de la segunda empresa en importancia (Sandoz). Poco más de una década después, Pioneer mantenía el liderazgo en volumen de ventas, pero aparecieron "nuevas" empresas, como Novartis, nacida en 1996 de la fusión de Sandoz y Ciba Geigy, ambas suizas, la cual se fusionó en diciembre de 1999 con Astra Zeneca, anglo-sueca, para formar Syngenta. De manera paralela, Monsanto comenzaba su larga trayectoria de fusiones y adquisiciones, pues desde fines de los ochenta y durante los noventa adquirió numerosas compañías involucradas en la producción de semillas y en biotecnología, entre las que destacan Dekalb en 1997 y Asgrow en 1998. Sin embargo, en 2005 hubo un fuerte reacomodo, pues Pioneer perdió su título de la compañía semillera más grande del mundo cuando Monsanto adquirió Seminis (la productora mundial de semillas de hortalizas y frutas) en enero de 2005 por 1,400 millones de dólares, con lo cual Monsanto se convirtió en la compañía de semillas con mayor volumen de ventas del mundo (Cuadro 1). Dentro de la estrategia comercial de estas empresas, el maíz juega un papel importante y al menos las cinco empresas de mayor volumen de venta en 2007, comercializan grandes volúmenes de esta especie. En el caso de Monsanto, su estrategia comercial en los últimos años se basa en los llamados maíces biotecnológicos (transgénicos). En ese mismo año, las empresas que comercializan maíces transgénicos tuvieron un volumen de ventas de 3200 millones de dólares, equivalente a $50 \%$ del mercado biotecnológico mundial (Clive, 2007).

\section{SITUACIÓN ACTUAL DE LA ACTIVIDAD SEMILLERA EN MÉXICO}

La industria de semillas en México se compone de: agricultores individuales, grandes empresas multinacionales, empresas privadas nacionales, y dependencias de investigación pública. El sector privado tiene $94 \%$ de la cuota de mercado, y el resto es del sector público (USDA, 2008).

Según datos del Directorio de Productores, Obtentores y Comercializadores de Semillas (SNICS, 2009), en México la producción de semilla global de los diversos cultivos está concentrada en dos regiones: a) en el norte del país, en los Estados de Sonora con 179 productores de semilla; Sinaloa, 82; Tamaulipas, 52; Coahuila, 45; Chihuahua, 44; y Baja California, 39; y b) en El Bajío, en los Estados de Guanajuato y Jalisco con 42 productores de semillas en cada caso.

Sin embargo, la producción de semilla certificada no ha sido suficiente para cubrir la demanda interna. La importación de semillas de alta calidad para siembra ha aumentado cada año (al menos 300 mil toneladas en 2008). Estados Unidos sigue siendo uno de los principales proveedores de semillas de flores y hortalizas para México, y se prevé que abastecerá casi $27 \%$ del total de las importaciones de semilla (USDA, 2008). En cuanto a maíz, no se tiene información de importación de semilla para siembra.

\section{Producción de semilla certificada de maíz en México}

De acuerdo con la información del SNICS (2010), la producción de semilla certificada de maíz en México, según el ciclo agrícola, se concentró de la siguiente manera:

Cuadro 1. Evolución de ventas de las empresas de semillas en años representativos de las últimas tres décadas.

\begin{tabular}{|c|c|c|c|c|c|c|c|}
\hline \multicolumn{2}{|c|}{1985} & \multicolumn{2}{|c|}{1996} & \multicolumn{2}{|c|}{2004} & \multicolumn{2}{|c|}{2007} \\
\hline Empresa & $\begin{array}{l}\text { Millones } \\
\text { USD }\end{array}$ & Empresa & $\begin{array}{l}\text { Millones } \\
\text { USD }\end{array}$ & Empresa & $\begin{array}{l}\text { Millones } \\
\text { USD }\end{array}$ & Empresa & $\begin{array}{l}\text { Millones } \\
\text { USD }\end{array}$ \\
\hline Pioneer & 735 & Pioneer & 1500 & Pioneer & 2600 & Monsanto & 4964 \\
\hline Sandoz & 290 & Novartis & 900 & Monsanto & 2277 & Pioneer & 3300 \\
\hline Dekalb & 201 & Limagrain & 650 & Syngenta & 1239 & Syngenta & 2018 \\
\hline Asgrow & 200 & Advanta & 460 & Limagrain & 1044 & Limagrain & 1226 \\
\hline Limagrain & 180 & Seminis & 375 & KWS AG & 622 & LandO'Lakes & 917 \\
\hline Nickerson & 175 & Takii & 320 & LandO'Lakes & 538 & KWS AG & 702 \\
\hline Takii & 175 & Sakata & 300 & Sakata & 416 & Bayer & 524 \\
\hline Ciba Geigy & 152 & KWS AG & 255 & Bayer & 387 & Sakata & 396 \\
\hline Sumatoria & 2108 & & 4760 & & 9123 & & 14047 \\
\hline
\end{tabular}

Fuente: Grupo ETC (2005, 2007, 2008), Le Buanec (2007) y Morales (2001). USD = Dólares americanos 
En los ciclos primavera-verano 2002 a 2008, $66 \%$ se produjo en Guanajuato, $11 \%$ en Jalisco y $9 \%$ en Querétaro. La empresa Monsanto produjo 75, 84 y $98 \%$, respectivamente, del total de la producción en esos estados.

En los ciclos otoño-invierno 2001-2002 a 2007-2008, 56 $\%$ se produjo en Sinaloa, $18 \%$ en Nayarit y $15 \%$ en Sonora. Monsanto representó 68, 85 y $71 \%$ respectivamente del total de la producción en estos estados.

En el año 2009 en México se sembraron aproximadamente 8 millones de hectáreas de maíz de las cuales se estima que 6.5 millones se sembraron con semilla nativa de polinización libre y 1.5 millones con semilla híbrida (SIAP, 2010). Si se considera únicamente la superficie sembrada con semilla híbrida y una densidad de siembra promedio de 30 kilogramos por hectárea, se necesitarían 45 mil toneladas de semilla certificada de maíz. De esa cantidad, aproximadamente $95 \%$ o sea 42 mil toneladas, son producidas por las grandes empresas transnacionales (Monsanto y Pioneer, principalmente) y el resto por las demás empresas (3 mil toneladas).

\section{DISCUSIÓN}

Después de un proceso de concentración, las tres empresas productoras y comercializadoras de semilla más importantes a nivel mundial son Monsanto, Pioneer y Syngenta. En México, estas tres empresas transnacionales atienden principalmente a la agricultura comercial, es decir, a los productores con grandes extensiones de tierra y con una mejor dotación de recursos tanto agroecológicos como tecnológicos, que tienen una orientación de producción para el mercado. Existe un segmento del mercado que no es de interés para este tipo de empresas, pues no cumple con el perfil de clientes establecido por ellas. Este segmento incluye, entre otros, a productores en regiones de secano o temporal medio a escaso cuyas necesidades de semilla certificada las atendía parcialmente la PRONASE.

Por lo anterior, se justifica y se propone el establecimiento de un mayor número de pequeñas empresas productoras de semilla a escala local y regional, pues según la información señalada se requerirían cerca de 3 mil toneladas de semilla para sembrar aproximadamente 100 mil hectáreas (con una densidad de $30 \mathrm{~kg}$ por hectárea). Al respecto, se ha reconocido que el desarrollo local debe basarse en diagnósticos y propuestas de manera participativa con los actores y considerar los recursos locales o particulares. En estos casos, la aplicación de resultados puede ser factible mediante la operación de microempresas (Pray y Ramaswami, 1991; Ostrom, 2009); tal puede ser la situación de los productores tradicionales para así disponer de semillas de calidad y abastecimiento oportuno. Es recomendable, además, que tales microempresas sean dirigidas por especialistas en la materia y asociaciones de productores, que tengan como objetivo atender a nichos de mercado específicos con características particulares, como son los productores de pequeña escala, con superficies de tierra menores de 5 hectáreas y para regiones agroecológicas especiales.

Para que las pequeñas y medianas empresas productoras de semilla tengan más posibilidades de éxito, se debe desarrollar todo un modelo y políticas (cadena de valor) que faciliten su conexión con otros aspectos relacionados que permita su funcionamiento y viabilidad a largo plazo. Por ejemplo, se requiere que las empresas productoras de semilla certificada de maíz tengan acceso fluido a fuentes de semilla original para iniciar el proceso de producción y su posterior comercialización. Es decir, fortalecer un sistema que comprenda y articule la generación de tecnología, vías de transferencia a los productores de semilla y su distribución a los productores agrícolas. Otro aspecto es el relacionado con la dinámica de la producción agrícola, en cuestiones de asesoría técnica y financiamiento para productores y con acceso garantizado al mercado de granos y alternativas de comercialización.

Con esto se desea destacar que hay necesidad de crear enlaces que ahora no se tienen, porque el esquema gubernamental no contempla esta estrategia como una alternativa viable. Por ello es necesario determinar las figuras o instituciones que se podrían constituir o fortalecer los elementos para asegurar el impacto de esta propuesta.

Por último, es necesario considerar la parte económica, pues entre más pequeña es la operación que se propone mayor es el gasto. Para ello se requieren estudios que demuestren la factibilidad económica de estas empresas, en los que se resalten los elementos que permitan desarrollar ventajas y capacidades para competir a nivel local y regional y de esta manera ofrecer a los productores agrícolas alternativas accesibles para adquirir semillas mejoradas.

\section{AGRADECIMIENTOS}

Al SNICS, por la información proporcionada.

\section{BIBLIOGRAFÍA}

Ayala-Garay O J, G García-de los Santos, A V Ayala-Garay, R Schwentesius-Rindermann (2006) Influencia de aspectos normativos en el desarrollo de la investigación, la enseñanza y la producción de semillas, el ejemplo del Colegio de Postgraduados. In: Memorias del Foro Nacional Agenda del Desarrollo 20062020. Políticas de Desarrollo Agropecuario, Forestal y Pesquero. R Schwentesius-Rindermann, M A Gómez-Cruz, A V AyalaGaray (eds) CIESTAAM-Universidad Autónoma Chapingo. 20-21 septiembre. México. pp:149-163. 
Clive J (2007) Global Status of Commercialized Biotech/GM Crops: 2007. International Service for the Acquisition of Agri-biotech Applications (ISAAA). Brief No. 37. New York, USA. 16 p.

Copeland L O, M B McDonald (2001) Principles of Seed Science and Technology. 4th ed. Kluwer Academic Publishers. Massachusetts, USA. $467 \mathrm{p}$.

DOF, Diario Oficial de la Federación (1961) Ley sobre Producción, Certificación y Comercio de Semillas. Secretaría de Agricultura y Ganadería. Poder Ejecutivo de la Federación. México, D.F. pp:2-8.

DOF, Diario Oficial de la Federación (1991) Ley sobre Producción, Certificación y Comercio de Semillas. Secretaría de Agricultura y Recursos Hidráulicos. Poder Ejecutivo de la Federación. México. D.F. pp:9-13.

Espinosa A, M Sierra, N Gómez (2003) Producción y tecnología de semillas mejoradas de maíz por el INIFAP en el escenario sin la PRONASE. Agron. Mesoam. 14:117-121.

Espinosa A, M Tadeo, A Turrent, N Gómez, M Sierra, A Palafox, F Caballero, R Valdivia, F A Rodríguez (2008) El potencial de las variedades nativas y mejoradas de maíz. Ciencias 92-93: 118-125.

Grupo ETC, Grupo de Acción sobre Erosión, Tecnología y Concentración (2005) Communiqué No. 90. Septiembre/ Octubre. Ottawa, Canadá. 13 p.

Grupo ETC, Grupo de Acción sobre Erosión, Tecnología y Concentración (2007) Las 10 Compañías de Semillas más Importantes del Mundo 2006. Ottawa, Canadá. 2 p.

Grupo ETC, Grupo de Acción sobre Erosión, Tecnología y Concentración (2008) Communiqué No. 100. ¿De Quién es la Naturaleza? El Poder Corporativo y la Frontera Final en el Mercantilismo de la Vida. Noviembre. Ottawa, Canadá. 52 p.

González A, J Islas, A Espinosa, J A Vázquez, S Wood (2008) Impacto Económico del Mejoramiento Genético del Maíz en México. Publicación Especial No. 25. INIFAP. México. 88 p.

Guillén-Pérez L A, C Sánchez-Quintanar, S Mercado-Domenech, H Navarro-Garza (2002) Análisis de atribución causal en el uso de semilla criolla y semilla mejorada de maíz. Agrociencia 36: 377387.

Jiménez-Sánchez L (1984) Las Ciencias Agrícolas y sus Protagonistas. Colegio de Postgraduados. Chapingo, México. 401 p.

Le Buanec B (2007) Evolution of the seed industry during the past three decades. ISTA News Bull. 134:6-10.

Morales C (2001) Las Nuevas Fronteras Tecnológicas: Promesas, Desafíos y Amenazas de los Transgénicos. CEPAL/Naciones Unidas. Serie Desarrollo Productivo 101. Santiago, Chile. 77 p.

Ostrom E (2009) A general framework for analyzing sustainability of social-ecological systems. Science 24:419-422.

Pray C E, Ramaswami B (1991) A Framework for Seed Policy Analysis. International Food Policy Research Institute. Washignton, USA. $42 \mathrm{p}$.
SIAP, Servicio de Información Agroalimentaria y Pesquera (2007) Situación Actual y Perspectivas del Maíz en México 1996 - 2012. Secretaría de Agricultura, Ganadería, Desarrollo Rural, Pesca y Alimentación (SAGARPA). México, D.F. 208 p.

SIAP, Servicio de Información Agroalimentaria y Pesquera (2010) Estadísticas de Producción de Semilla del Año Agrícola 2009 Disponible en: http://www.siap.gob.mx/aagricola_siap/icultivo/ index.jsp (Julio 2011).

SNICS, Servicio Nacional de Inspección y Certificación de Semillas (2009) Directorio de Productores, Obtentores y Comercializadores de Semillas. SNICS-SAGARPA, México D.F. 338 p.

SNICS, Servicio Nacional de Inspección y Certificación de Semillas (2010) Base de Datos de Producción de Semilla Certificada de Maíz y Empresas Productoras a Nivel Nacional, Años 2002-2008. SNICS-SAGARPA. México, D.F. 14 p.

SNICS, Servicio Nacional de Inspección y Certificación de Semillas (2011a) Estadísticas de Producción de Semilla de los Años Agrícolas 1988-1992. Disponible en: http://snics.sagarpa.gob. mx/certificacion/estadisticas/Paginas/AA-1988-1992.aspx (Julio 2011).

SNICS, Servicio Nacional de Inspección y Certificación de Semillas (2011b) Estadísticas de Producción de Semilla de los Años Agrícolas 1993-1997. Disponible en: http://snics. sagarpa.gob.mx/certificacion/estadisticas/Paginas/AA-1993-1997. aspx (Julio 2011).

SNICS, Servicio Nacional de Inspección y Certificación de Semillas (2011c) Estadísticas de Producción de Semilla de los Años Agrícolas 1998-2002. Disponible en: http://snics.sagarpa.gob. mx/certificacion/estadisticas/Paginas/AA\%201998-02.aspx (Julio 2011).

SNICS, Servicio Nacional de Inspección y Certificación de Semillas (2011d) Estadísticas de Producción de Semilla de los Años Agrícolas 2003-2007. Disponible en: http://snics.sagarpa.gob. $\mathrm{mx} /$ certificacion/estadisticas/Paginas/AA-2003-2007.aspx (Julio 2011).

SNICS (Servicio Nacional de Inspección y Certificación de Semillas) (2011e) Estadísticas de Producción de Semilla de los Años Agrícolas 2008-2009. Disponible en: http://snics.sagarpa.gob. $\mathrm{mx} /$ certificacion/estadisticas/Paginas/AA-2008-2009.aspx （Julio 2011).

Tansey G, T Rajotte (2009) El Control Futuro de los Alimentos. Ed. Mundi-Prensa. Madrid, España. 312 p.

Turrent-Fernández A (2009) El potencial productivo de maíz. Ciencias 92-93:126-129.

USDA, United States Department of Agriculture-Foreign Agricultural Service (2008) Global Agriculture Information Network (GAIN) Report: Mexico Planting Seeds Annual. Washington, USA. 8 p. 